12

\title{
Расчет мультипольной цилиндрической электростатической системы
}

\author{
(C) Е.М. Виноградова, Н.В. Егоров, А.В. Старикова, М.И. Вараюнь
}

Санкт-Петербургский государственный университет,

198504 Санкт-Петербург, Россия

e-mail: vincat2008@yandex.ru, robby7@mail.ru

(Поступило в Редакцию 13 июля 2016 г.)

Представлены результаты математического моделирования мультипольной электронно-оптической системы. Электроды системы представляют собой четное число одинаковых частей одного кругового цилиндра бесконечной длины, разрезанного параллельно образующей цилиндра. Для нахождения распределения потенциала граничная задача для уравнения Лапласа решено методом разделения переменных в полярных координатах. Все геометрические размеры системы и число электродов являются параметрами задачи.

DOI: 10.21883/JTF.2017.05.44454.1990

\section{Введение}

Электростатические мультипольные линзы широко используются в различных современных электронновакуумных приборах формирования и транспортировки пучков заряженных частиц в ускорительной технике, электронной микроскопии, например, растровых электронных микроскопах, масс-спектрометрах, для корректировки сферической и хроматической аберрации объектива, для фокусировки или ускорения пучков заряженных частиц с малым значением первеанса [1-4].

Электростатические квадрупольные линзы часто предпочтительнее магнитных при фокусировки пучков заряженных частиц средних энергий. Кроме того, данный вид линз используется для работы с ионными пучками, так как оптическая сила электростатической линзы не зависит от массы заряженной частицы, в отличии от магнитной. В зависимости от назначения прибора могут

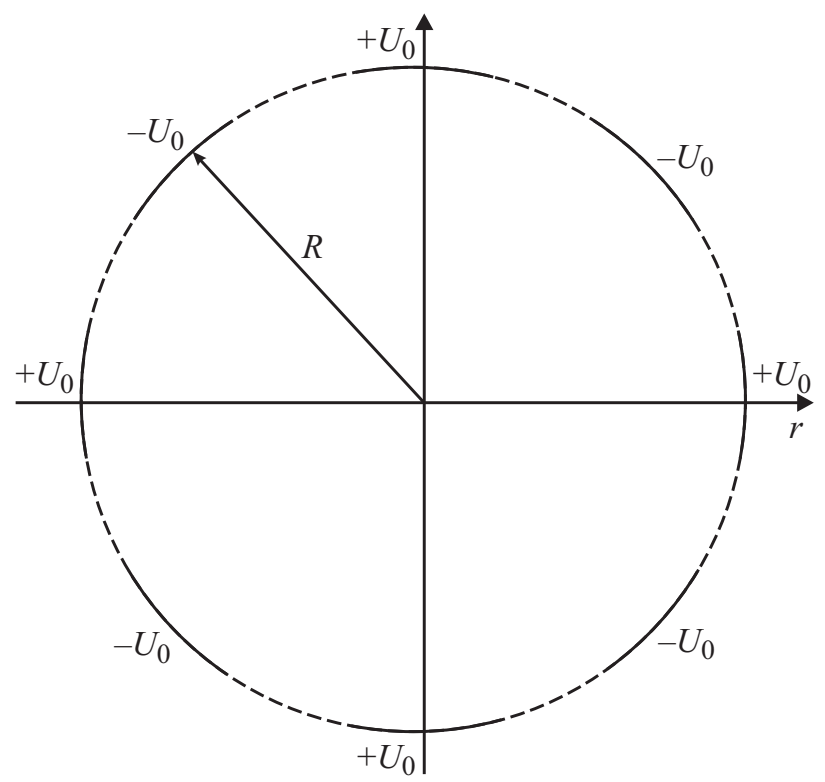

Рис. 1. использоваться как одиночные линзы, так и системы корректирующих линз [5].

В последнее время значительный прогресс в высокоточном производстве и компьютерном управлении технологий производства электронно-оптических приборов привел к созданию квадрупольно-октупольных систем линз. Гексапольный корректор широко используется для сканирующего туннельного электронного микроскопа $[6-8]$.

Для расчета дипольных, квадрупольных, секступольных, октупольных электростатических полей проводятся как экспериментальные исследования [9], так и теоретические [10,11].

В настоящей работе моделируется электростатическая мультипольная линза, состоящая из $2 n$ электродов с одинаковыми геометрическими параметрами, каждый из которых представляет собой часть кругового цилиндра бесконечной длины, и симметрично расположенных относительно $n$ плоскостей симметрии, пересекающихся по оси данного цилиндра радиуса $R_{1}$. На электродах задан постоянный, одинаковый по модулю потенциал $U_{0}$, чередующийся по знаку на соседних электродах. В качестве примера мультипольной линзы на рис. 1. представлено сечение октупольной системы в плоскости, перпендикулярной оси цилиндра.

\section{Математическая модель}

В силу того, что $2 n$ электродов мультипольной линзы представляют собой четное число одинаковых частей одного кругового цилиндра бесконечной длины, разрезанного параллельно образующей, с чередующимися по знаку потенциалами на соседних электродах, данная система имеет $n$ плоскостей симметриии $n$ плоскостей антисимметрии.

Для вычисления распределения электростатического потенциала используется метод разделения переменных [12-14]. Зададим полярные координаты $(r, \alpha)$ на плоскости так, чтобы поле, создаваемое данной 


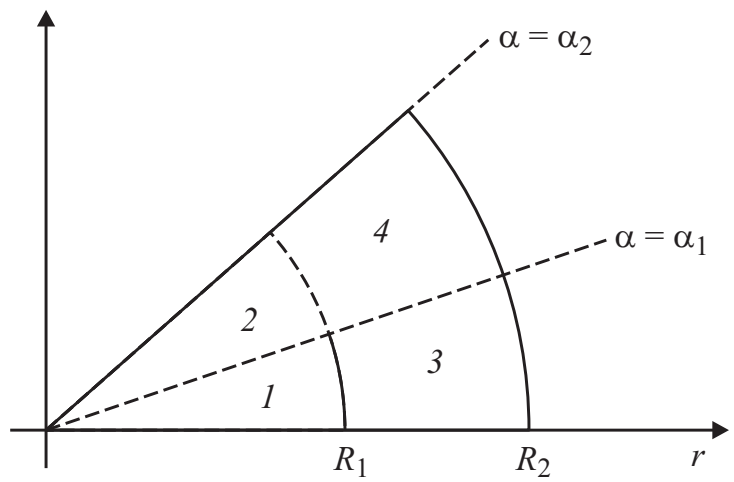

Рис. 2.

мультипольной системой электродов, было симметрично относительно аксиальных плоскостей $\alpha=(\pi k) / n$ и антисимметрично относительно аксиальных плоскостей $\alpha=\pi / 2 n+(\pi k) / n, k \in Z$. Не ограничивая общность задачи, зададим внешнюю границу рассматриваемой области $r=R_{2}$ с нулевым потенциалом. Таким образом, достаточно рассмотреть сектор $0 \leq \alpha \leq \alpha_{2}, 0 \leq r \leq R_{2}$, $\alpha_{2}=\pi / 2 n$ (рис. 2).

Параметры системы: $R_{1}$ - радиус цилиндра, задающего электроды системы, $R_{2}$ - радиус внешней границы системы, $\left(R_{1}, \alpha_{1}\right)$ - координата края электрода, $2 n-$ число электродов, $U_{0}$ - потенциал электрода.

Функция распределения электростатического потенциала $U(r, \alpha)$ удовлетворяет уравнению Лапласа с граничными условиями

$$
\begin{gathered}
\frac{1}{r} \frac{\partial}{\partial r}\left(r \frac{\partial U}{\partial r}\right)+\frac{1}{r^{2}} \frac{\partial^{2} U}{\partial \alpha^{2}}=0, \\
U\left(R_{1}, \alpha\right)=U_{0}, \quad 0 \leq \alpha \leq \alpha_{1}, \\
U\left(R_{2}, \alpha\right)=0, \quad 0 \leq \alpha \leq \alpha_{2}, \\
U\left(r, \alpha_{2}\right)=0, \quad 0 \leq r \leq R_{2}, \\
\left.\frac{\partial U}{\partial \alpha}\right|_{\alpha=0}=0, \quad 0 \leq r \leq R_{2},
\end{gathered}
$$

где $\alpha=\alpha_{2}=\pi / 2 n-$ плоскость антисимметрии.

\section{Решение задачи}

Для решения граничной задачи $(1)-(5)$ разобьем всю внутреннюю область системы $\left(0 \leq r \leq R_{2}, 0 \leq \alpha \leq \alpha_{2}\right)$ на четыре области:

$$
\begin{aligned}
& 1-\left(0 \leq \alpha \leq \alpha_{1}, \quad 0 \leq r \leq R_{1}\right) \\
& 2-\left(\alpha_{1} \leq \alpha \leq \alpha_{2}, \quad 0 \leq r \leq R_{1}\right) \\
& 3-\left(0 \leq \alpha \leq \alpha_{1}, \quad R_{1} \leq r \leq R_{2}\right) \\
& 4-\left(\alpha_{1} \leq \alpha \leq \alpha_{2}, \quad R_{1} \leq r \leq R_{2}\right)
\end{aligned}
$$

Распределение потенциала $U(r, \alpha)=U_{i}(r, \alpha)$ $(i=\overline{1,4})$ для каждой из указанных областей можно представить в виде: в первой области

$$
\begin{aligned}
U_{1}(r, \alpha)= & \sum_{n=0}^{\infty} a_{n}\left(\frac{r}{R_{1}}\right)^{\mu_{n}} \cos \left(\mu_{n} \alpha\right) \\
& +\int_{0}^{\infty} b(\lambda) \frac{\operatorname{ch}(\lambda \alpha)}{\operatorname{ch}\left(\lambda \alpha_{1}\right)} \sin \left(\lambda \ln \frac{r}{R_{1}}\right) d \lambda,
\end{aligned}
$$

во второй области

$$
\begin{aligned}
& U_{2}(r, \alpha)=\sum_{k=1}^{\infty} c_{k}\left(\frac{r}{R_{1}}\right)^{v_{k}} \sin \left(v_{k}\left(\alpha_{2}-\alpha\right)\right) \\
& +\int_{0}^{\infty} b(\lambda) \frac{\operatorname{sh}\left(\lambda\left(\alpha_{2}-\alpha\right)\right)}{\operatorname{sh}\left(\lambda\left(\alpha_{2}-\alpha_{1}\right)\right)} \sin \left(\lambda \ln \frac{r}{R_{1}}\right) d \lambda,
\end{aligned}
$$

в третьей области

$$
\begin{gathered}
U_{3}(r, \alpha)=\sum_{n=0}^{\infty} a_{n} \frac{\left(\frac{r}{R_{2}}\right)^{\mu_{n}}-\left(\frac{R_{2}}{r}\right)^{\mu_{n}}}{\left(\frac{R_{1}}{R_{2}}\right)^{\mu_{n}}-\left(\frac{R_{2}}{R_{1}}\right)^{\mu_{n}}} \cos \left(\mu_{n} \alpha\right) \\
+\sum_{m=1}^{\infty} d_{m} \frac{\operatorname{ch}\left(v_{m} \alpha\right)}{\operatorname{ch}\left(v_{m} \alpha_{1}\right)} \sin \left(\beta_{m} \ln \frac{r}{R_{2}}\right),
\end{gathered}
$$

в четвертой области

$$
\begin{aligned}
& U_{4}(r, \alpha)=\sum_{n=0}^{\infty} c_{k} \frac{\left(\frac{r}{R_{2}}\right)^{v_{k}}-\left(\frac{R_{2}}{r}\right)^{v_{k}}}{\left(\frac{R_{1}}{R_{2}}\right)^{v_{k}}-\left(\frac{R_{2}}{R_{1}}\right)^{v_{k}}} \sin \left(\left(v_{k}\left(\alpha_{2}-\alpha\right)\right)\right. \\
& +\sum_{m=1}^{\infty} d_{m} \frac{\operatorname{sh}\left(\beta_{m}\left(\alpha_{2}-\alpha\right)\right)}{\operatorname{sh}\left(\beta_{m}\left(\alpha_{2}-\alpha_{1}\right)\right)} \sin \left(\beta_{m} \ln \frac{r}{R_{2}}\right),
\end{aligned}
$$

где

$$
\mu_{n}=\frac{\pi(2 n+1)}{2 \alpha_{1}}, \quad v_{k}=\frac{\pi k}{\alpha_{2}-\alpha_{1}}, \quad \beta_{m}=\frac{\pi m}{\ln \frac{R_{1}}{R_{2}}}
$$

- собственные значения,

$$
a_{0}=U_{0} \frac{2}{\pi}, \quad a_{n}=U_{0} \frac{(-1)^{n} 4}{\pi(2 n+1)} \quad(n \geq 1)
$$

- коэффициенты разложения в ряд функции Фурье, задаваемой граничным условием (2). Распределение электростатического потенциала в виде (6)-(9) удовлетворяет уравнению Лапласа (1) и заданным граничным значениям (2)-(5). Непрерывность распределения потенциала на границах раздела областей $1-2,2-4$, 3-4 тождественно удовлетворяется из разложений по собственным функциям (6)-(9).

Условия непрерывности нормальной составляющей вектора электрического поля на границе раздела областей $1-2,2-4,3-4$ имеют вид

$$
\left.\frac{\partial U_{1}}{\partial \alpha}\right|_{\alpha=\alpha_{1}}=\left.\frac{\partial U_{2}}{\partial \alpha}\right|_{\alpha=\alpha_{1}}, \quad 0 \leq r \leq R_{1},
$$




$$
\begin{array}{ll}
\left.\frac{\partial U_{2}}{\partial r}\right|_{r=R_{1}}=\left.\frac{\partial U_{4}}{\partial r}\right|_{r=R_{1}}, \quad \alpha_{1} \leq \alpha \leq \alpha_{2}, \\
\left.\frac{\partial U_{3}}{\partial \alpha}\right|_{\alpha=\alpha_{1}}=\left.\frac{\partial U_{4}}{\partial \alpha}\right|_{\alpha=\alpha_{1}}, \quad R_{1} \leq r \leq R_{2},
\end{array}
$$

Выполнение условий (12)-(14) приводит к следующей системе уравнений относительно неизвестных наборов коэффициентов $b(\lambda), c_{k}, d_{m}$, входящих в функции распределения потенциала (6)-(9):

$$
\begin{gathered}
b(\lambda)\left[\operatorname{th} \lambda \alpha_{1}+\operatorname{cth} \lambda\left(\alpha_{2}-\alpha_{1}\right)\right]+\frac{2}{\pi} \sum_{k=1}^{\infty} c_{k}(-1)^{k} \frac{v_{k}}{\lambda^{2}+v_{k}^{2}} \\
=\frac{2}{\pi} \sum_{n=0}^{\infty} a_{n}(-1)^{n} \frac{\mu_{n}}{\lambda^{2}+\mu_{n}^{2}}, \\
\int_{0}^{\infty} b(\lambda)(-1)^{k} \frac{\lambda}{v_{k}^{2}+\lambda^{2}} d \lambda-c_{k} \frac{\alpha_{2}-\alpha_{1}}{1-\left(R_{1} / R_{2}\right)^{2 v_{k}}} \\
+\sum_{m=1}^{\infty} d_{m}(-1)^{m+1} \frac{\beta_{m}}{v_{k}^{2}+\beta_{m}^{2}}=0, \\
\sum_{k=1}^{\infty} c_{k}(-1)^{k} \frac{v_{k}}{\beta_{m}^{2}+v_{k}^{2}}+d_{m}(-1)^{m+1}\left[\operatorname{th} \beta_{m} \alpha_{1}\right. \\
\left.+\operatorname{cth} \beta_{m}\left(\alpha_{2}-\alpha_{1}\right)\right] \ln \sqrt{R_{2} / R_{1}}=\sum_{n=0}^{\infty} a_{n}(-1)^{n} \frac{\mu_{n}}{\beta_{m}^{2}+\mu_{k}^{2}} .
\end{gathered}
$$

Решение системы уравнений (15)-(17) определяет значения неизвестных коэффициентов в разложении функций распределения потенциала (6)-(9) во всей области исследуемой системы.

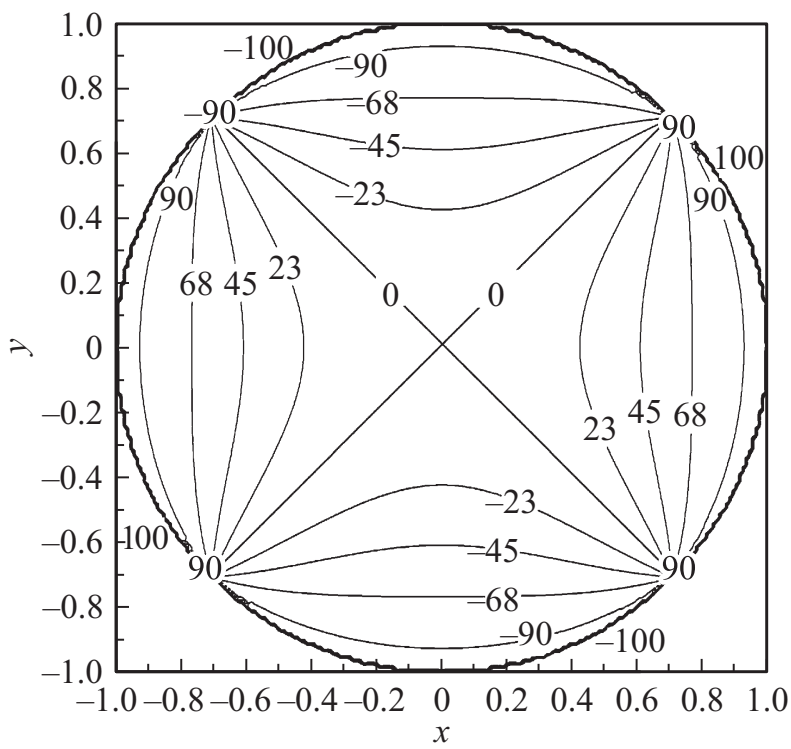

Puc. 3.

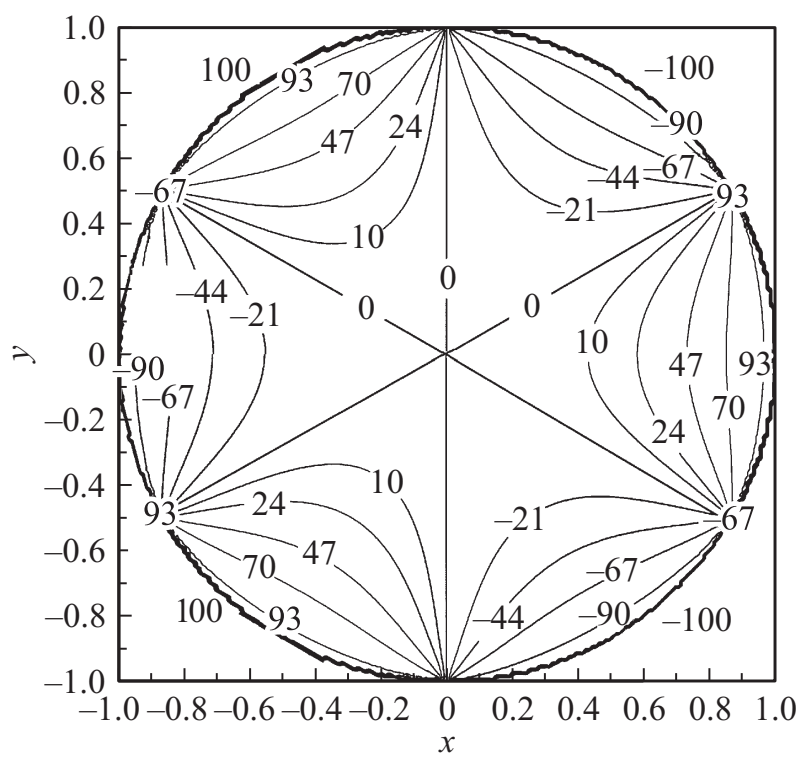

Pис. 4.

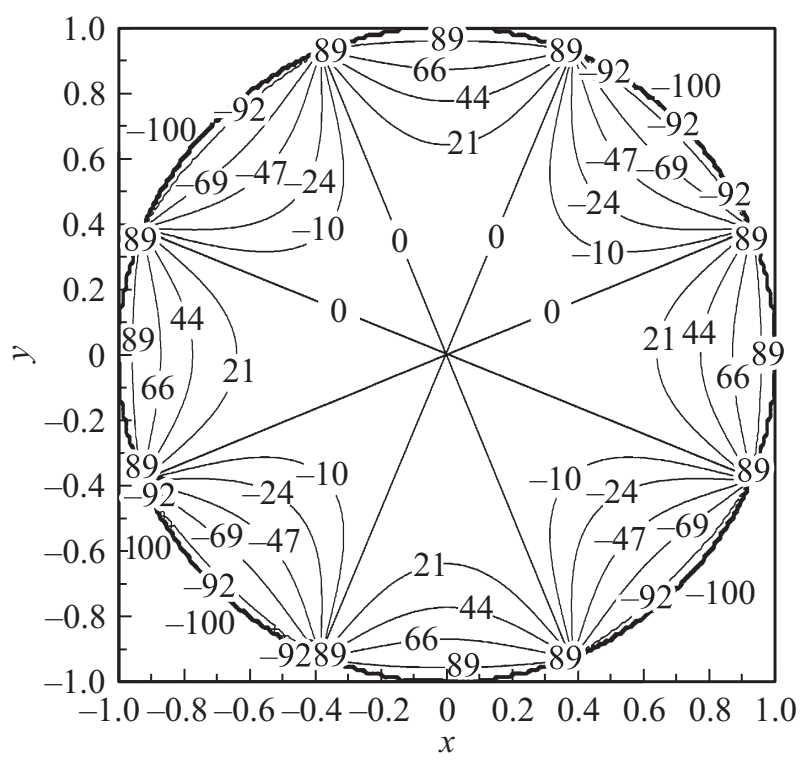

Рис. 5.

\section{Результаты численных расчетов}

В случае, если $\alpha_{1}=\alpha_{2}=\pi / 2 n$, решение граничной задачи (1)-(5) значительно упрощается, так как вся внутренняя область системы представляет собой область $1-\left(0 \leq \alpha \leq \alpha_{1} ; 0 \leq r \leq R_{1}\right)$. Распределение электростатического потенциала имеет вид

$$
U_{1}(r, \alpha)=\sum_{n=0}^{\infty} a_{n}\left(\frac{r}{R_{1}}\right)^{\mu_{n}} \cos \left(\mu_{n} \alpha\right),
$$

где $\mu_{n}$ и $a_{n}$ вычисляются по формулам (10), (11) соответственно. 


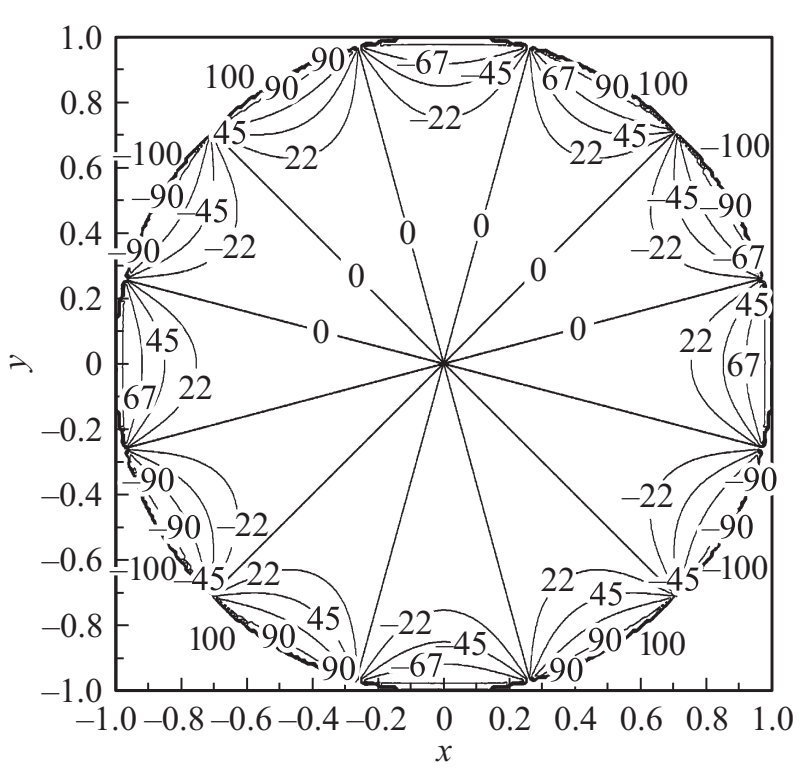

Рис. 6.

При расчете распределения электростатического потенциала по формуле (18) использовались следующие параметры в безразмерных величинах: $R_{1}=1, U_{0}=100$.

На рис. 3 представлены эквипотенциальные линии распределения потенциала во всей области квадрупольной системы при $n=2$.

На рис. 4 представлены эквипотенциальные линии распределения потенциала во всей области секступольной системы при $n=3$.

На рис. 5 представлены эквипотенциальные линии распределения потенциала во всей области октупольной системы при $n=4$.

На рис. 6 представлены эквипотенциальные линии распределения потенциала во всей области додекапольной системы при $n=6$.

\section{Заключение}

В настоящей работе найдено распределение потенциала во всей области электростатической мультипольной системы, состоящей из произвольного четного числа электродов с одинаковыми геометрическими параметрами, каждый из которых представляет собой часть кругового цилиндра бесконечной длины.

Для решения граничной задачи $(1)-(5)$ использовался метод разделения переменных в полярных координатах. Для случая $\alpha_{1} \neq \alpha_{2}$ распределение электростатического потенциала представлено в виде разложений по собственным функциям (6)-(11), а определение неизвестных коэффициентов в разложениях потенциала сведено к решению системы алгебраических уравнений (15)-(17). Для случая $\alpha_{1}=\alpha_{2}=\pi / 2 n$ аналитическое решение граничной задачи имеет явный вид - (10), (11), (18) и в соответствии с полученными формулами представлены эквипотенциальные линии распределения потенциала для четырех мультипольных систем (квадрупольной, секступольной, октупольной, додекапольной).

Все геометрические размеры системы и значения потенциалов на электродах представляют собой параметры задачи.

\section{Список литературы}

[1] Овсянникова Л.П., Фишкова Т.Я., Петров И.А.. // ЖТФ. 2001. Т. 71. Вып. 12. С. 62-65.

[2] Овсянникова Л.П., Фишкова Т.Я. // ЖТФ. 2001. Т. 71. Вып. 5. С. 96-99.

[3] Баранова Л.A., Read F.H., Cubric D. // ЖТФ. 2009. Т. 79. Вып. 7. С. 85-91.

[4] Елизаров A.A., Сорокин E.A. // Радиотехника и электроника. 2012. Т. 57. Вып. 11. С. 1212-1220.

[5] Baranova L.A., Read F.H. // Optik. 2001. Vol. 112. N 3. P. $131-138$.

[6] Tamura K., Okayama S., Shimizu R. // J. Electron. Microsc. 2010. Vol. 59. N 3. P. 197-206.

[7] Baranova L.A., Read F.H. // Int. J. Mass Spectr. 1999. Vol. 189. P. 19-26.

[8] Xiu K., Murray Gibson J. // Optik. 2001. Vol. 112. N 11. P. $521-530$.

[9] Бадалян Г.В., Айвазян Г.М., Нерсесян Я.Д. // ЖТФ. 2014. Т. 84. Вып. 1. С. $127-138$.

[10] Спивак-Лавров И.Ф.. // ЖТФ. 1999. Т. 69. Вып. 3. С. 1-9.

[11] Виноградова Е.М., Листрукова А.В. // Вестн. СПбГУ. Cер. 10: Прикладная математика, информатика, процессы управления. 2016. Вып. 1. С. 19-27.

[12] Виноградова Е.М., Егоров Н.В., Мутул М.Г., Шэнь ЧэЧоу. // ЖТФ. 2010. Т. 80. Вып. 5. С. 1-4.

[13] Виноградова Е.М., Егоров Н.В., Мутул М.Г., Кримская К.А. // ЖТФ. 2008. Т. 78. Вып. 8. С. 128-131.

[14] Egorov N.V., Vinogradova E.M. // Vacuum. 2004. Vol. 72. N 2. P. $103-111$. 